\title{
Wireless stepper motor control and optimization based on robust control theory
}

\author{
Waleed Khalid Al-Azzawi \\ Medical Technical College, AI-Farahidi University, Baghdad, Iraq
}

\begin{tabular}{l} 
Article Info \\
\hline Article history: \\
Received Apr 9, 2020 \\
Revised Jan 10, 2021 \\
Accepted Feb 9, 2021 \\
\hline
\end{tabular}

\section{Keywords:}

Model reference control

Robust control

Stepper motors

Uncertainty parameters

\begin{abstract}
Stepper motors are broadly utilized in actual systems, which are marked by non-linear parameters such as internal, external noises and uncertainties from wireless network. As well, a suitable controller is required when the problem is to track the target signal. In this paper, robust controller based on model reference are investigated to wireless control and optimize position and time in stepper motors. The core impression to build a robust controller is to use a model reference control system. Furthermore, simulations are implemented to control stepper motor position and time in two cases: first, when the wireless network without any delay and packet dropout. Second, uncertain equations when the wireless network with time delays and packet dropout. Simulation results demonstrate that proposed controller has achieved and enhanced the performance in tracking and robustness.
\end{abstract}

This is an open access article under the $\underline{C C B Y-S A}$ license.

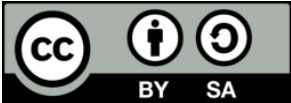

\section{Corresponding Author:}

Waleed Khalid Al-Azzawi

Medical Technical College

AI-Farahidi University, Baghdad, Iraq

Email: waleed.khalid@alfarahidiuc.edu.iq; waleedki@yahoo.com

\section{INTRODUCTION}

Hybrid stepper motor is a non-linear motor that has been investigated to rotate in specific angular position. Any stepper motors (SMs) require conventional controllers for position and speed control. Thus, SMs are currently utilized in manufacturing and different engineering applications. Recently, SM is widely used in several motion control applications for example robotics, printers, and discrete control circuits. Currently, numerous methods have been presented for positioning, speed control and obtained of appropriate controller with wireless network in SM. It is important that a complex controller will be required due to nonlinear structure of SM and wireless network, while output tracking problem is characterized. In recent decades, adaptive algorithms have been applied to hybrid SM increased [1, 2]. Otherwise, the other techniques such as PID controller [3, 4] and robust controller have been improved particularly for uncertain parameters systems [5-7]. Similarly, numerous of the research focus on system identification and control based on intelligent controller such as neural networks, genetic algorithms, and fuzzy logic [8-10].

The neural network is design based on back-propagation model and trained for SM identification. In addition, integration of fuzzy logics and adaptive control is used to obtained required control signal [11-13]. Furthermore, used genetic algorithm and adaptive control is built for SM identification [14-16]. In contrast, this paper designs a model reference robust controller for uncertain parameters in wireless network such as time delay, minimizing distance and time in SM. In this paper, we use true-time tools based model reference controller system in order to create a robust controller able to remove and minimize all uncertain parameters of the wireless network SM model. It can be seen from the block diagram in Figure 1 that in wireless networked control system the closed-loops are closed via wireless communication networks. The supplement of the wireless network in the feedback control loop makes the analysis and design of systems more difficult 
than the conventional structural model. The wireless network control system can create undependable and time delay-dependent stages of service in terms of, for example, time delays, jitter, or packet dropouts. The quality of service (QoS) can improve the real-time wireless network control system performance, however, the wireless network performance is still subject to frequency interference, bandwidth limitation, to aggressive flows. It is also noteworthy that protocols providing the quality of service are not common in all real-time networks [2].

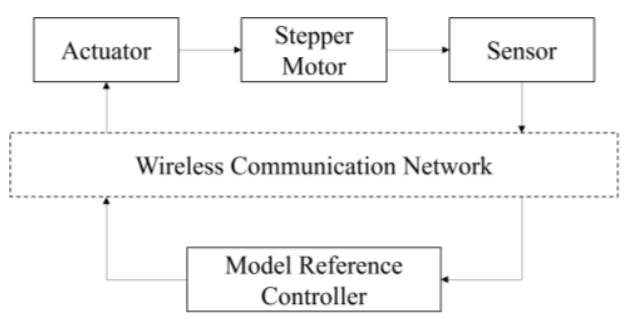

Figure 1. Typical setup of wireless SM

\section{MATHEMATICAL MODEL OF SM}

The mathematical models are derived according to the following assumptions: Mutual inductances are ignored, minimum variation of self-inductance with mechanical angle, fluxes are assumed to be sinusoidal around mean value, neglected all torques values.

\subsection{Electrical model}

$$
\begin{aligned}
i_{a} & =\left[v_{a}-R i_{a}+k_{m} w \sin \left(N_{r} \theta\right)\right] / L \\
i_{b} & =\left[v_{b}-R i_{b}+k_{m} w \cos \left(N_{r} \theta\right)\right] / L
\end{aligned}
$$

where, $i_{a}$ and $i_{b}$ are armatures and phase current respectively, $v_{a}$ and $v_{b}$ are armature and phase voltage, $N_{r}$ is the number of teeth, $R$ is resistance, $k_{m}$ is motor torque, $w$ is angular speed of motor, and $L$ is inductance.

\subsection{Mechanical model}

$$
\begin{aligned}
& \dot{w}=\frac{1}{J}\left[-k_{m} i_{a} \sin \left(N_{r} \theta\right)+k_{m} i_{b} \cos \left(N_{r} \theta\right)-B w-T_{L}-k_{D} \sin \left(4 N_{r} \theta\right)\right] \\
& \dot{\theta}=w
\end{aligned}
$$

where, $\mathrm{J}$ is motor moment of inertia, $B$ is friction viscous factor, $T_{L}$ is load torque, $k_{D}$ is drive torque, and $\dot{\theta}$ is motor position.

$$
u_{r}=\frac{v_{a}}{L} \text { and } u_{b}=\frac{v_{b}}{L}
$$

By defining $x=\left[\frac{x_{1}}{x_{2}}\right]=\left[\frac{\dot{w}}{\dot{\theta}}\right]$ the control system can be written as follows.

$$
\begin{aligned}
& \dot{x}_{1}=x_{2} \cdots \cdots \cdots \\
& \dot{x}_{2}=\frac{k_{m} L}{R J}\left(u_{r}-\frac{R x_{2}}{L} u_{d}\right)
\end{aligned}
$$

\subsection{Robust controller design}

A robust model reference controller proposed design to investigate two objectives in the presence of uncertainty and without it. Firstly, system output tracks the decided reference value. Secondly, system to be stable and all uncertain parameters with limit time.

To design such a controller, the following assumptions will be considered. Reference vector $\theta_{d}=$ $\left[\theta_{d}, \dot{\theta}_{d}\right]$ is defined such that $\left\|\dot{\theta}_{d}\right\|<\theta_{1}$ and $\left\|\theta_{d}\right\|<\theta_{0}$; where, $\theta_{1}$ and $\theta_{0}$ are known positive constants. Take in your account that we want to calculate the sign of system function in order to design the robust controller. The sign of states must be constant over all domains, while $\alpha$ is defined to determine the sign of system

Wireless stepper motor control and optimization based on robust control theory (Waleed Khalid Al-Azzawi) 
function and its value is selected according to system requirement. Also, $u_{r}$ and $u_{d}$ are considered as the system inputs and defined as follows.

$$
u_{r}=\alpha, u_{d}=\alpha U
$$

where, $U$ is control signal generated by control system.

\section{SIMULATION RESULTS}

In this section, we apply robust control design theory to state equation which obtained from section 2. Also, we compared the system performance when used traditional control and robust control in output signal tracking control. For this purpose, we required to use system (5) in SM block of block-diagram which has been shown in Figure 1. For the purpose of simulation, we use the following values for the system parameters, obtained from [14]:

$$
\begin{aligned}
& R=100 \Omega, L=0.001 \mathrm{H}, B=0.001 N_{m \frac{s e c}{r a d}}, J=57 \times 10^{-7} \mathrm{Kgm}^{2}, N_{r}=50, \text { and } \\
& k_{m}=114 \times 10^{-3} N_{m} / A
\end{aligned}
$$

It must be noted that all torques values has been neglected in (1). The purpose of control design is to have the output of rotor position tracks the desirable path shown in Figure 2 with minimum error. To demonstration the efficiency of proposed controller, in addition to certain model of the system, the experiments have been implemented in the presence of uncertainty. The model reference error between the wireless SM output and the robust model reference controller output is used as the error training signal. The procedure is illustrated in Figure 3. Tracking performance for traditional and robust control system, under certain model condition, is shown in Figure 4.

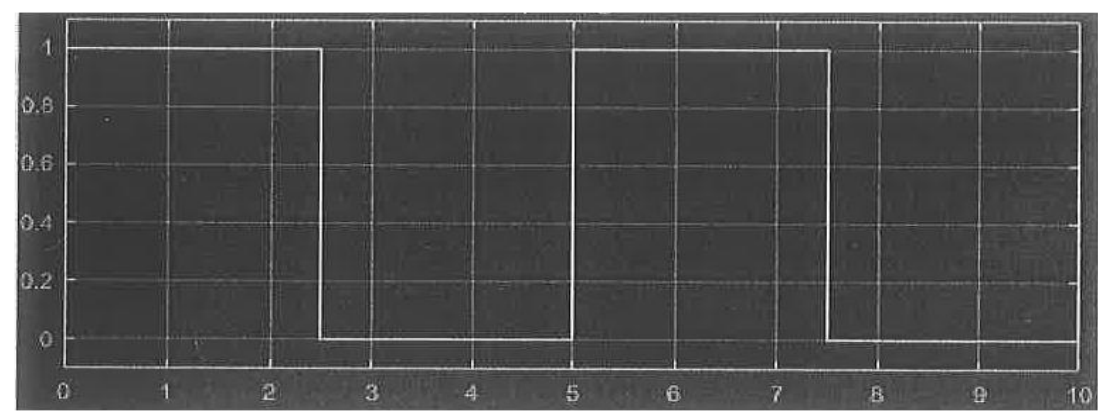

Figure 2. Desired motor input

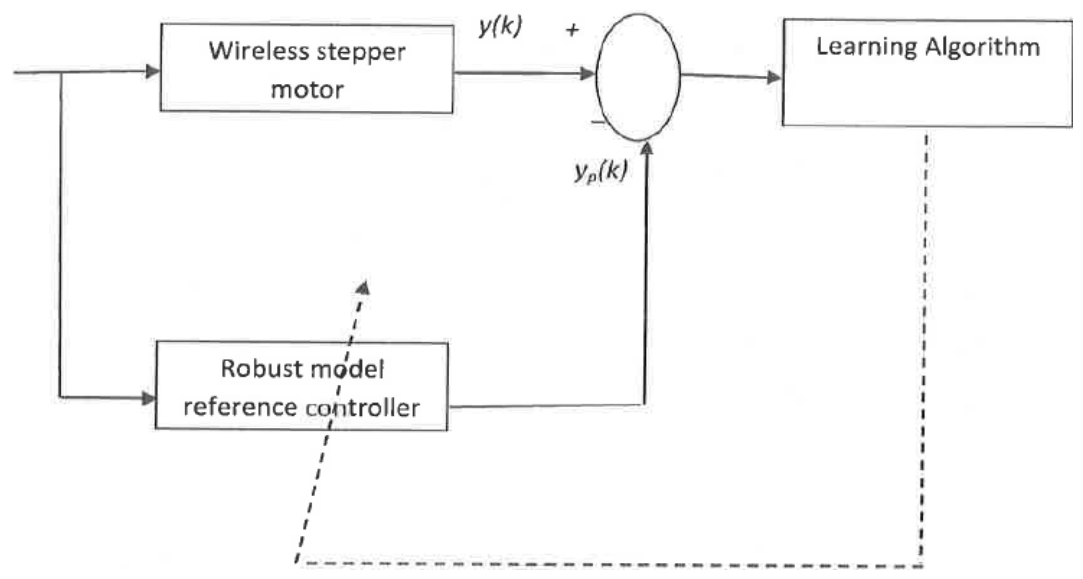

Figure 3. Procedure of error learning algorithm 
It must be seen that traditional controller modelling is designed according to [16]. As well, model factors are constant in both traditional and robust model reference control system during simulation process. It is observed from Figure 4 that the proposed robust model reference controller reaches to the desirable output faster than traditional controller. As shown in Figure 4, time constant of traditional controller and proposed robust model reference controllers are 0.029 and 0.017 , respectively. Furthermore, simulation results with the existence of system factors uncertainty have been shown in Figure 5. It must be noted that uncertainty has been applied to rotor, load inertia, and viscous friction coefficient of the SMs [3]. Similarly, a random disturbance has been applied to load and viscous friction coefficient in scale of $58 \times 10^{-9}$ and $2 \times 10^{-5}$, respectively. In Figure 5, uncertainty is applied to load and viscous friction coefficient, respectively, and tracking performance is compared for both traditional controller and robust model reference controllers. It is observable that proposed robust model reference controller has better performance in tracking control signal and also faster response rather than traditional controller. Also, proposed controller is more robust against modifications and uncertain parameters of the system model.

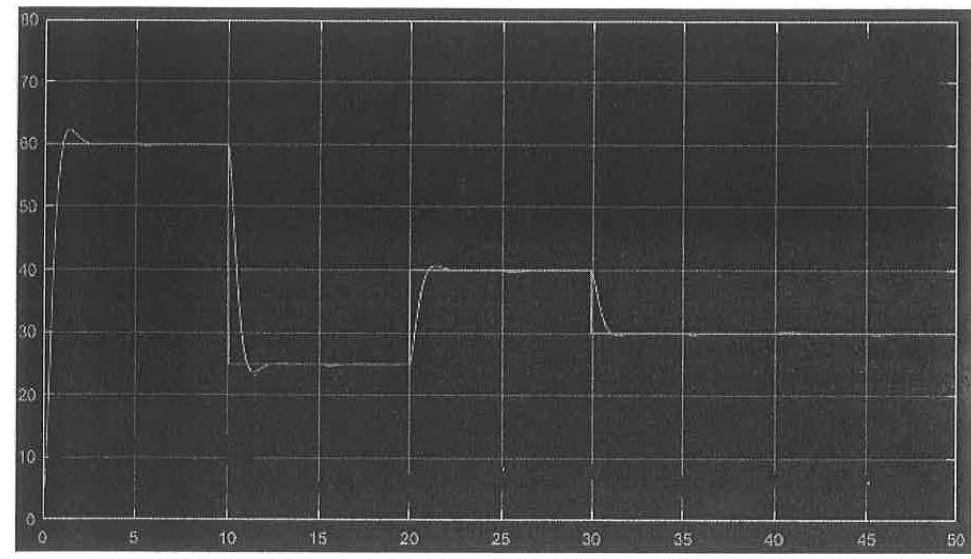

Figure 4. Wireless SM response with robust controller without uncertain parameters

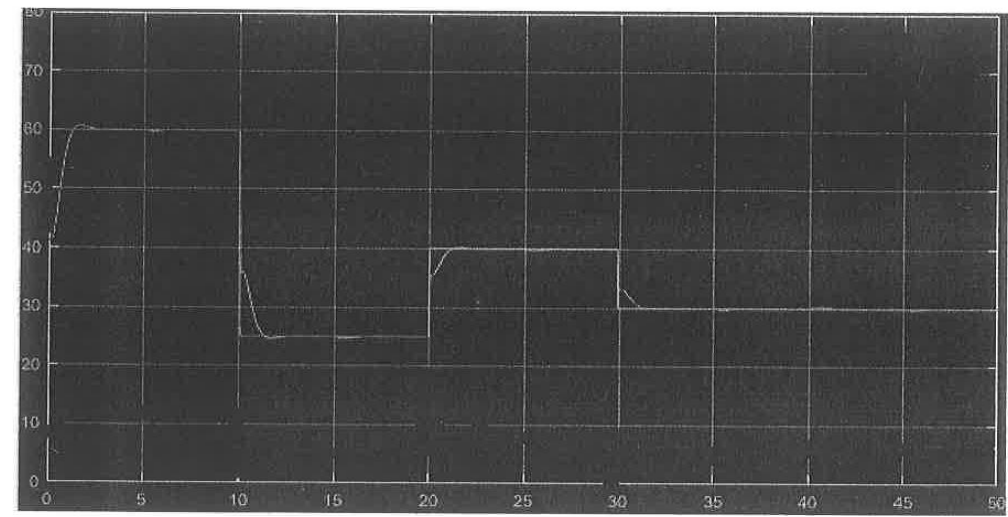

Figure 5. Wireless SM response with robust controller with uncertain parameters

\section{CONCLUSION}

Based on simulation results, it was detected that proposed robust model reference controller has enhanced the tracking control signal and more robustness response in comparison with traditional controller in the presence of both uncertainties. Actually, using of uncertain signals applied to system model and robust controller methods to model nonlinear and indefinite functions, results in handling uncertainties much better than the traditional controller. Thus, the effect of uncertainties removed or becomes minimum. Finally, it was presented that robust controller provides high performance in presence of uncertain signal and the simulation results demonstrated the main objective of the proposed controller that was accurate output position control. 


\section{REFERENCES}

[1] V. Del Toro, Electric machines and power systems. NJ: Prentice-Hall, 1985.

[2] M. S. M. Elksasy and H. H. Gad, "A new technique for controlling hybrid stepper motor through modified PID controller," International Journal of Electrical \& Computer Sciences IJECS-IJENS, vol. 10, no. 2, pp. 28-35, 2014.

[3] A. S. Kumar and V. Jayalakshmi, "Modelling of speed control based on digital PID controller for pm stepper motor," International Journal of Pure and Applied Mathematics, vol. 116, no. 19, pp. 137-142, 2017.

[4] J. G. Vlachogiannis and K. Y. Lee, "Economic load dispatch-A comparative study on heuristic optimization techniques with an improved coordinated aggregation-based PSO," in IEEE Transactions on Power Systems, vol. 24, no. 2, pp. 991-1001, May 2009, doi: 10.1109/TPWRS.2009.2016524.

[5] Q. Wu and C. Shao, "Novel hybrid sliding-mode controller for direct torque control induction motor drives," in 2006 American Control Conference, 2006, pp. 5, doi: 10.1109/ACC.2006.1656640.

[6] R. Majumder, B. Chaudhuri, H. El-Zobaidi, B. Pal, and I. Jaimoukha, "LMI approach to normalised $\mathscr{H} \infty 0^{\circ}$ loopshaping design of power system damping controllers," IEE Proceedings - Generation, Transmission and Distribution, vol. 152, no. 6, p. 952, 2005.

[7] A. Rahim and E. P. Nowicki, "Performance of a grid-connected wind generation system with a robust susceptance controller," Electric Power Systems Research, vol. 81, no. 1, pp. 149-157, 2011.

[8] T. Radpukdee and P. Jirawattana, "Uncertainty learning and compensation: An application to pressure tracking of an electro-hydraulic proportional relief valve," Control Engineering Practice, vol. 17, no. 2, pp. 291-301, 2009.

[9] X. Huang and L. Shi, "Simulation on a Fuzzy-PID Position Controller of the CNC Servo System," in Sixth International Conference on Intelligent Systems Design and Applications, 2006, pp. 305-309, doi: 10.1109/ISDA.2006.237.

[10] E. Kilic, M. Dolen, H. Caliskan, A. B. Koku, and T. Balkan, "Pressure prediction on a variable-speed pump controlled hydraulic system using structured recurrent neural networks," Control Engineering Practice, vol. 26, pp. 51-71, 2014

[11] G. Feng, "Position control of a PM stepper motor using neural networks," in Proceedings of the 39th IEEE Conference on Decision and Control (Cat. No.00CH37187), 2000, pp. 1766-1769 vol. 2, doi: 10.1109/CDC.2000.912117.

[12] M. D. Minkov, J. L. Rodgerson, and R.G. Harly, "Adaptive neural speed controller of a dc motor," Electric Power Systems Research, vol. 47, no. 2, pp. 123-132, 1998.

[13] K. Nouri, R. Dhaouadi, and N. B. Braiek, "Adaptive control of a nonlinear de motor drive using recurrent neural networks," Applied Soft Computing, vol. 8, no. 1, pp. 371-382, 2008

[14] W. A. Bedwani and O. M. Ismail, "Genetic optimization of variable structure PID control systems," in Proceedings ACS/IEEE International Conference on Computer Systems and Applications, Beirut, Lebanon, 2001, pp. 27-30, doi: 10.1109/AICCSA.2001.933947.

[15] M. R. Zaidan, "Impact of artificial neural network for DC motor speed control over the conventional controller," Journal of Engineering and Applied Sciences, vol. 13, no. 21, pp. 9156-9163, 2018.

[16] B. M. Zaineb, A. Aicha, B. H. Mouna, and S. Lassaad, "Speed control of DC motor based on an adaptive feed forward neural IMC controller," in 2017 International Conference on Green Energy Conversion Systems (GECS), Hammamet, 2017, pp. 1-7, doi: 10.1109/GECS.2017.8066278. 